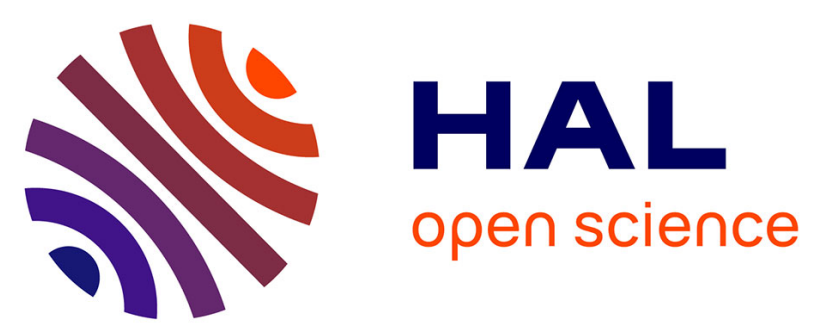

\title{
Determination of hardness and elastic modulus inverse pole figures of a polycrystalline commercially pure titanium by coupling nanoindentation and EBSD techniques
}

\author{
C. Fizanne-Michel, M. Cornen, P. Castany, I. Péron, T. Gloriant
}

\section{To cite this version:}

C. Fizanne-Michel, M. Cornen, P. Castany, I. Péron, T. Gloriant. Determination of hardness and elastic modulus inverse pole figures of a polycrystalline commercially pure titanium by coupling nanoindentation and EBSD techniques. Materials Science and Engineering: A, 2014, 613, pp.159-162. 10.1016/j.msea.2014.06.098 . hal-01153829

\section{HAL Id: hal-01153829}

https://hal-univ-rennes1.archives-ouvertes.fr/hal-01153829

Submitted on 4 Oct 2018

HAL is a multi-disciplinary open access archive for the deposit and dissemination of scientific research documents, whether they are published or not. The documents may come from teaching and research institutions in France or abroad, or from public or private research centers.
L'archive ouverte pluridisciplinaire HAL, est destinée au dépôt et à la diffusion de documents scientifiques de niveau recherche, publiés ou non, émanant des établissements d'enseignement et de recherche français ou étrangers, des laboratoires publics ou privés. 


\title{
Determination of hardness and elastic modulus inverse pole figures of a polycrystalline commercially pure titanium by coupling nanoindentation and EBSD techniques
}

\author{
C. Fizanne-Michel, M. Cornen*, P. Castany, I. Péron, T. Gloriant \\ UMR CNRS 6226 Institut Sciences Chimique de Rennes, INSA Rennes, 20 avenue des Buttes \\ de Coësmes, 35708 Rennes Cedex 7, France
}

*Corresponding author: Dr Marilyne Cornen

Mail : marilyne.cornen@insa-rennes.fr

Tel : +(0)33223238239

\begin{abstract}
Nanoindentation and electron backscattered diffraction (EBSD) techniques were used to study the correlation between crystallographic orientations and mechanical properties of individual grains in CP-Ti polycrystalline samples. Therefore, novel experimental protocol and data analyses procedure have been implemented in order to obtain elastic modulus and hardness inverse pole figures (EIPF and HIPF). It has been demonstrated that the hardness varies significantly with orientation, and that the elastic modulus appears less sensitive than hardness to grain orientation.
\end{abstract}

Keywords: Titanium alloys, electron backscattered diffraction (EBSD), Nanoindentation, Hardness, Elastic modulus.

\section{Introduction and objective}

The nanoindentation technique is well known to be very efficient to investigate the local mechanical properties of materials. Indeed, this technique is being used increasingly to measure hardness, elastic modulus, creep parameters and residual stresses thanks to highresolution load control and displacement sensing capabilities [1]. This technique is particularly useful to characterize microscale heterogeneities in materials or local mechanical 
properties at the grain scale in polycrystalline alloys. For this latter, crystal orientation versus hardness and elastic modulus at the grain scale can be of great interest in order to understand and to predict texture or anisotropy influences on macroscopic mechanical properties of engineering alloys. However, only few studies concerning hardness and elastic modulus measurements with regards to the grain orientation for polycrystalline alloys exist in literature [2-9]. This study was focused on the $\alpha$ phase of titanium, a widespread phase in titanium alloys used typically in highly oxidizing and corrosive environments, in the aerospace industry, in chemical industry, and for various biomedical applications [10].

In this paper, both an experimental protocol and a data analyses procedure for determining the correlation between grain orientation and theirs properties (hardness, elastic modulus) are described. During the experiments, nanoindentation and electron backscattered diffraction (EBSD) techniques were used to investigate the anisotropy of the indentation behaviour of individual grains, that constitute the microstructure of a polycrystalline $\alpha$ titanium which possesses an hexagonal closed packed crystallographic structure (hcp). Using these methods, it is thus possible to determine the relationship between grain orientations and hardnesses $\left(\mathrm{H}_{\mathrm{hkl}}\right)$ or elastic modulii $\left(\mathrm{E}_{\mathrm{hkl}}\right)$ by drawing inverse pole figures, which represent simultaneously the level of hardness or elastic modulus and the crystallographic orientation. The specific aim of this study has been to achieve hardness inverse pole figures (HIPF) and elastic modulus inverse pole figures (EIPF) with a sufficiently high statistic in order to cover the whole crystallographic orientations.

\section{Materials and methods}

The study was conducted on the commercially pure titanium (CP-Ti, grade 2) possessing the following nominal composition in weight $\%: 0,03 \% \mathrm{~N}, 0,10 \% \mathrm{C}, 0,015 \% \mathrm{H}, 0,3 \% \mathrm{Fe}, 0,25 \% \mathrm{O}$ and the balance Ti. Slices were cut from the as-received $20 \mathrm{~mm}$ diameter bar. In order to obtain the combination of an optimal grain size and a wide range of crystallographic orientations, the following thermo-mechanical protocol was realized: first a cold rolling deformation at room temperature until $50 \%$ of reduction with a final thickness of $\mathrm{e}_{\mathrm{f}}=3 \mathrm{~mm}$ was applied. Then, a recrystallization treatment at $870^{\circ} \mathrm{C}$ for $2 \mathrm{~h}$ under high vacuum was performed. In order to represent as well as possible all the crystallographic orientations contained in the sample (and thus cover the whole fundamental triangle in IPF), analyses were performed both on the rolling and the transverse planes because it was observed a different texture in those two planes. Indeed, the rolling plane owns crystallographic orientations 
mainly near the [0001] direction and the transverse plane owns crystallographic orientations close to $[10 \overline{10}$ and $[2 \overline{1} \overline{10}$. Thus, combination of both planes analyses allows a better statistic.

Prior to the electron backscattered diffraction and nanoindentation characterizations, each specimen surface was carefully prepared by polishing with several SiC papers of decreasing grid size followed by a final polishing with colloidal silica suspension of $0,02 \mu \mathrm{m}$. Finally, a chemical etching of few seconds in a $5 \% \mathrm{HNO}_{3}, 5 \% \mathrm{HF}$ and $90 \% \mathrm{H}_{2} \mathrm{O}$ solution (vol.\%) was performed to remove the work hardening layer and reveal the microstructure. Note that a high quality final surface finish is absolutely essential for both EBSD measurements and the subsequent nanoindentation experiments.

The electron backscattered diffraction analyses were carried out on a JEOL JSM 6400 scanning electron microscope equipped with a TSL EBSD system. Then, in the grains previously located through EBSD maps, nanoindentations measurements were carried out using a CSM nanoindentation tester (NHT CSM). Two indenters were used: a $10 \mu \mathrm{m}$ radius spherical diamond tip on one hand and a modified Berkovich diamond tip $\left(\alpha=65,3^{\circ}\right)$ on the other hand. Load controlled indentation tests were led at room temperature, and the analyses were conducted using the Oliver and Pharr method [1]. Nanoindentations were performed with a maximum load of $50 \mathrm{mN}$ and $100 \mathrm{mN}$ for the Berkovich and the spherical indenters, respectively. During the entire loading and unloading cycle, the applied load and the displacement of the indenter were recorded continuously. Hardness and elastic modulus values have been extracted from the unloading curves according to the equivalent indenter method [1]. For each tip, multiple indentations were performed on the rolling and transverse planes, up to a total of 100 nanoindentation tests for each plane. Indents were performed in the middle of grains in order to reduce as much as possible the influence of adjacent grains or grain boundaries, thus approximating as well as possible the behavior of a single crystal. Other characterizations at a greater scale have also been performed, such as microhardness and tensile tests, in order to compare the hardness and the elastic modulus values obtained at the microscopic and macroscopic scales. Microhardness tests were performed on a Mitutuyo machine equipped with a Vickers diamond tip. The tensile tests were carried out with an Instron 3369 machine up to the rupture of the sample at a strain rate of $10^{-4} \mathrm{~s}^{-1}$. For the tests, flat specimens with a section of $3 \times 0.7 \mathrm{~mm}$ and a gage length of $15 \mathrm{~mm}$ were machined 
before the recrystallization annealing. All tensile tests were performed with the tensile direction parallel to the rolling direction.

\section{Results and discussion}

As mentioned before, nanoindentation measurements correlated with the EBSD cartographies allow drawing inverse pole figures for hardness (HIPF) and for elastic modulus (EIPF). The aim of this representation is to permit to see at one glance both the level of hardness (or elastic modulus) and the orientation of one individual grain, drawn in the fundamental triangle. For this methodology, several EBSD inverse pole figure (IPF) cartographies of the polycrystalline microstructure were achieved to cover the orientations of a wide area of the sample, and nanoindentation measurements were conducted in smaller regions (from all parts of the large area), containing several grains, either with one tip or the other (only one kind of indenter by cartography). An example of a typical cartography obtained by EBSD technique is presented in Figure 1a where it can be observed the typical equiaxed $\alpha$-grain microstructure with a grain size of few tens $\mu \mathrm{m}$. The different grain orientations in the map are determined by a colour code related to the fundamental triangle of the hexagonal crystallographic structure of CP-Ti (Fig. 1b). To exemplify how to obtain a hardness inverse pole figure from this material, the 2 grains (G1 and G2) are chosen in the EBSD map (Fig. 1a), and then located in the fundamental triangle shown in Figure 1b. In those grains, a nanoindentation test has been performed, with one of the indentation tips mentioned previously (Fig 1c left). Note that the dimension of indents has never been higher than $1 \mu \mathrm{m}$ in width in this study. Then, a specific hardness colour code was established for the different values obtained from each grain (Fig 1c right). In the fundamental triangle, the grains G1 and G2 can now be highlighted by colored spots giving information of the hardness values determined (Fig 1d). So, by combination of these two informations, hardness and crystallographic orientation, the hardness inverse pole figure (HIPF) is then obtained. The same protocol has been applied to obtain an elastic modulus inverse pole figure (EIPF). In total, approximately 200 grains were characterized to obtain both HIPF and EIPF using both Berkovich and spherical tips in this study.

HIPF and EIPF results obtained with the two kinds of indentation tips are presented in figures 2 and 3 respectively. For each HIPF and EIPF, average values were assessed, and compared with literature firstly and with other mechanical testing secondly ${ }^{\text {Tab. } 1}$. Our average values for HIPF are found to be in the same order of magnitude to the ones obtained by Mante et al. [5], 
who measured an average hardness of $2,11 \pm 0,12 \mathrm{GPa}$ for a polycrystalline titanium sample (Berkovich tip, $\mathrm{F}_{\max }=5-150 \mathrm{mN}$ ). In the present study, the mean hardness of the alpha titanium were 2,9 $\pm 0,4 \mathrm{GPa}$ and 2,8 $\pm 0,6 \mathrm{GPa}$ with Berkovich and spherical tip, respectively (Table 1). Concerning EIPF results, the same authors [5] have measured an average elastic modulus of $128 \pm 7 \mathrm{GPa}$. Britton et al. [6] have measured Young's modulus values lying between 125 and $150 \mathrm{GPa}$ (Berkovich tip, $\mathrm{h}_{\max }=1 \mu \mathrm{m}$ ). These values are also very similar to those obtained in the present work since a mean elastic modulus of $130 \pm 7 \mathrm{GPa}$ and $140 \pm$ 10GPa were evaluated from Berkovich and spherical tip, respectively (Table 1). The average values calculated from EIPF and HIPF are close to literature, thus validating the methodology used here. One important point is that in the present study the whole fundamental triangle has been investigated, on the contrary of most previous works [6][8][13].

With the idea to compare the results achieved by nanoindentation to those measured by classical mechanical tests, a characterization was also performed on CP-Ti by microhardness and tensile tests. The results are presented in Table 1. A difference of $10 \%$ is observed between our results obtained by nanoindentation and the ones obtained by microindentation. In the same way, as regards to the tensile test, we noticed a difference of about $12 \%$ between the modulus measured at the macroscale and the one assessed at nanoscale. It can be seen that globally hardness and Young's modulus values determined by nanoindentation are overestimated compared to those obtained at the micro-macroscopic scale, which is consistent with the literature [11] [14]. Qian et al. [11] have observed that the nanoindentation hardness is $10-30 \%$ higher than microhardness. The main reason was attributed to the analyses methods: to estimate the hardness, the contact area is needed. But the achievement of this area is different for the nano/micro methods (projected area is calculated for nanoindentation [15][16] and residual area is directly measured for microindentation). If the area deduced from analysis of the load-displacement curves underestimates the true contact area, this leads to overestimations of both the hardness and the elastic modulus. So, taking this fact into account, it can be said that a good agreement has been obtained here between nano-scale and micro-scale experiments.

Drawing inverse pole figures with hardness or elastic modulus scales allows the observation in polycrystalline $\mathrm{CP}-\mathrm{Ti}$ of the correlation between the crystallographic orientation of a chosen single grain and its mechanical properties. To make easier the interpretation of the results, inverse pole figures have been divided into 4 parts, depending on the disorientation 
angle of each grain from simple directions. As it can be seen from Figure 2 no significant trends were found for Young's modulus values as a function of the crystallographic orientation. It means that whatever the crystallographic orientation of the grain is, all the values of the elastic modulus (in the range measured in the set of experiment) have equal probability. Similar results in polycrystalline samples have been highlighted by Merson and al. [7] and Roth and al. [17]. On the figure the visible outcome is that all the different colors of the modulus scale are mixed all over the fundamental triangle. However, the statistical variation of values is observed to be higher when the Berkovich tip was used. One reason for this fact is that the Berkovich tip doesn't allow to probe the elastic properties of the materials properly, because its salient tip plasticizes rapidly the indented area [12]. So a wide dispersion can be obtained in the results. On the contrary, there is a lower disparity in the EIPF obtained with the spherical tip. This low variation of modulus is consistent with the one found in the Britton and al. work [6] in which a difference of $20 \mathrm{GPa}$ was observed when moving away from the basal plane to the prismatic plane (along one side of the fundamental triangle only). The observed orientation dependence of hardness is also consistent with results obtained using tensile tests on single crystals [18] or on strongly textured polycrystals [19] [20], which evidenced a continuous decrease of the mechanical resistance when the tensile axis deviated from the [0001] axis.

The great statistics of our study has allowed to visualize that this trend is not only limited to one side of the fundamental triangle, but extends to the entire inverse pole figure. In contrast to the elastic modulus observations, a significant tendency was found between the hardness values of individual grains and their specific orientation. A recent study [2] has shown that the elastic modulus appears to be more sensitive to its neighboring than the hardness, implying that a difference in hardness is often observed whereas in modulus no trend is seen. As it can be seen on Figure 3, a clear rainbow appears in the fundamental triangle, showing a decrease of the hardness when the grains disorientation from [0001] increases. This effect is visible in both sets of experiments (Berkovich and Spherical). Indeed, nanoindentation tests in grains almost parallel to the basal plane, which means close to the [0001] direction, give the highest hardness values, lying in the ranges of 330-365 HV for Berkovich tip and 370-415 HV for the spherical tip (Figure 3). These trends demonstrate that the hardness of individual grains is strongly dependent on the orientation of the stress axis. These results are in good agreement with the literature $[6,7,8]$ where the basal planes were shown to be the hardest, with hardness values decreasing as the stress axis deviates from the [0001] direction. 


\section{Conclusion}

To summarize, this study examined the effect of crystallographic orientation on mechanical properties of individual grains, in a polycrystalline commercially pure titanium. We used EBSD technique to provide a mapping of crystallographic orientations of different polycrystalline areas of a sample and then nanoindentation technique to probe the mechanical properties of each grain spotted on the orientation maps. For a better understanding, and a highlighted visual effect, the results have been presented as "inverse pole figures in hardness" (HIPF) and "inverse pole figures in elastic modulus" (EIPF). It has been proved that the hardness varies significantly with orientation. On the contrary, the evolution of the elastic modulus appears less sensitive to grain orientation. The nanoindentation measurements carried out in present work show that hardnesses of grains presenting a basal plane possess higher values than grains presenting a prismatic plane of about $100 \mathrm{HV}$ with the Berkovich tip and about $140 \mathrm{HV}$ with the spherical tip, respectively.

\section{References}

[1] A.C. Fischer-Cripps, Nanoindentation, third ed., Springer, New-York, 2011.

[2] C. Tromas, J.C. Stinville, C. Templier, P. Villechaise, Acta Mater. 60 (2012) 1965-1973.

[3] A. Lodh, I. Samajdar, R. Tewari, D. Srivastava, G.K. Dey, P. Pant, Mater. Sci. Forum 702-703 (2012) 311-314.

[4] T. Miura, K. Fujii, K. Fukuya, K. Takashima, J. Nucl. Mater. 417 (2011) 984-987.

[5] F.K. Mante, G.R. Baran, B. Lucas, Biomaterials 20 (1999) 1051-1055.

[6] T.B. Britton, H. Liang, F.P.E. Dunne, A.J. Wilkinson, Proc. R. Soc. A466 (2010) 695-719.

[7] E. Merson, R. Brydson, A. Brown, J. Phys. Conference Series 126 (2008).

[8] C. Zambaldi, Y. Yang, T.R. Bieler, D. Raabe, J. Mater. Res. 27 (2012) 356-367.

[9] J. Kwon, M.C. Brandes, P. Sudharshan Phani, A.P. Pilchak, Y.F. Gao, E.P. George, G.M. Pharr, M.J. Mills, Acta Mater. 61 (2013) 4743-4756.

[10] D. Banerjee, J.C. Williams, Acta Mater. 61 (2013) 844-879.

[11] L. Qian, M. Li, Z. Zhou, H. Yang, X. Shi, Surf. Coat. Tech. 195 (2005) 264-271.

[12] R.A. Mirshams, R.M. Pothapragada, Acta Mater. 54 (2006) 1123-1134.

[13] G.B. Viswanathan, E. Lee, D.M. Maher, S. Banerjee, H.L. Fraser, Acta Mater. 53 (2005) 5101-5115. 
[14] A. Bolshakov and G.M. Pharr, Journal of Materials Research / Volume 13 / Issue 04 / 1998, pp 1049-1058.

[15] W.C. Oliver, G.M. Pharr, J. Mater. Res. 19 (2004) 3-20.

[16] M. Troyon, L. Huang, Surface and Coatings technology 201 (2006) 1613-1619.

[17] Roth and al., Materials Science \& Engineering A 596 (2014) 236-243.

[18] Churchman A.T., Proceedings of the Royal Society of London, vol 226, p216, 1954.

[19] Zarkades A., Larson F.R., The Science, Technology and Application of Titanium, Pergamon Press, Oxford, UK (1970) p.933.

[20] M.C. Brandes, M. Baughman, M.J. Mills, J.C. Williams, Materials Science and Engineering: A, Volume 551, 2012, p13-18.

Table 1. Comparison of nanoindentation results to classical mechanical tests.

\begin{tabular}{lcc}
\hline & $\mathrm{H}$ & $\mathrm{E}$ \\
\hline Nanoindentation Berkovich & $\begin{array}{c}2,9 \pm 0,4 \mathrm{Gpa} \\
270 \pm 40 \mathrm{HV}\end{array}$ & $130 \pm 7 \mathrm{Gpa}$ \\
\hline Nanoindentation Spherical & $\begin{array}{r}2,8 \pm 0,6 \mathrm{GPa} \\
290 \pm 60 \mathrm{HV}\end{array}$ & $140 \pm 10 \mathrm{Gpa}$ \\
\hline Microindentation & $250 \pm 20 \mathrm{HV}$ & - \\
\hline Tensile test & - & $119 \pm 17 \mathrm{GPa}$ \\
\hline
\end{tabular}


(a)

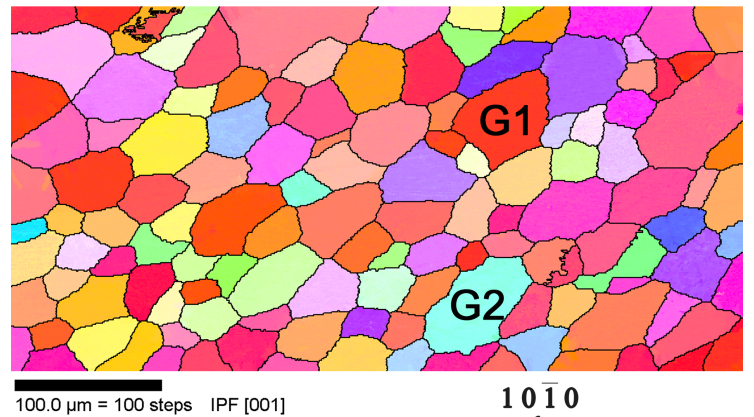

(b)

(c)
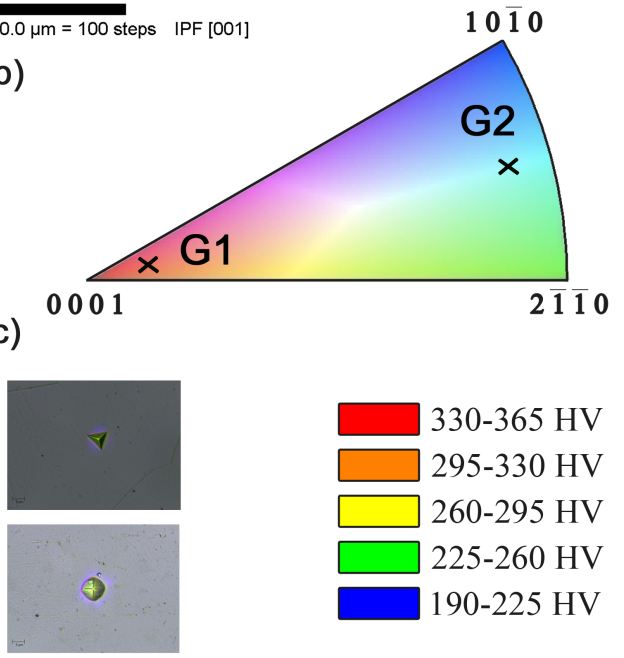

(d)

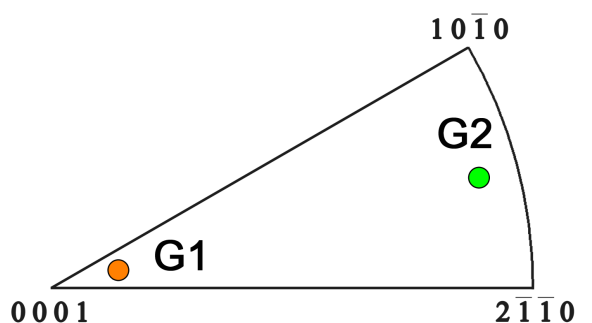

Figure 1. Methodology to obtain elastic modulus and hardness inverse pole figures. 

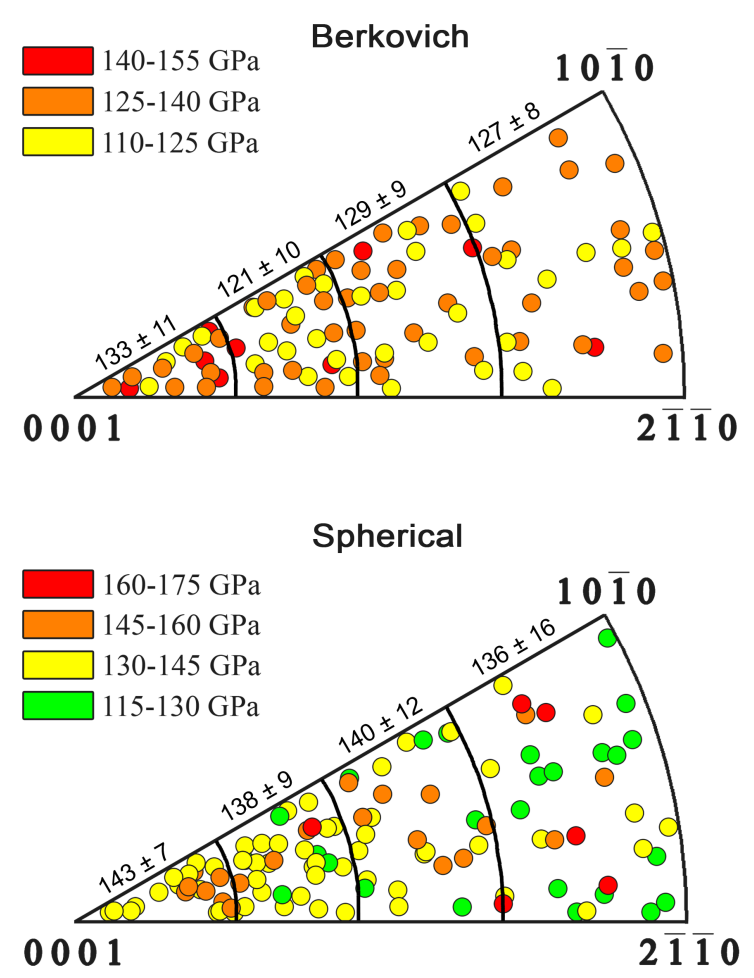

Figure 2. Elastic modulus inverse pole figures (EIPF) with both spherical and Berkovich tips.
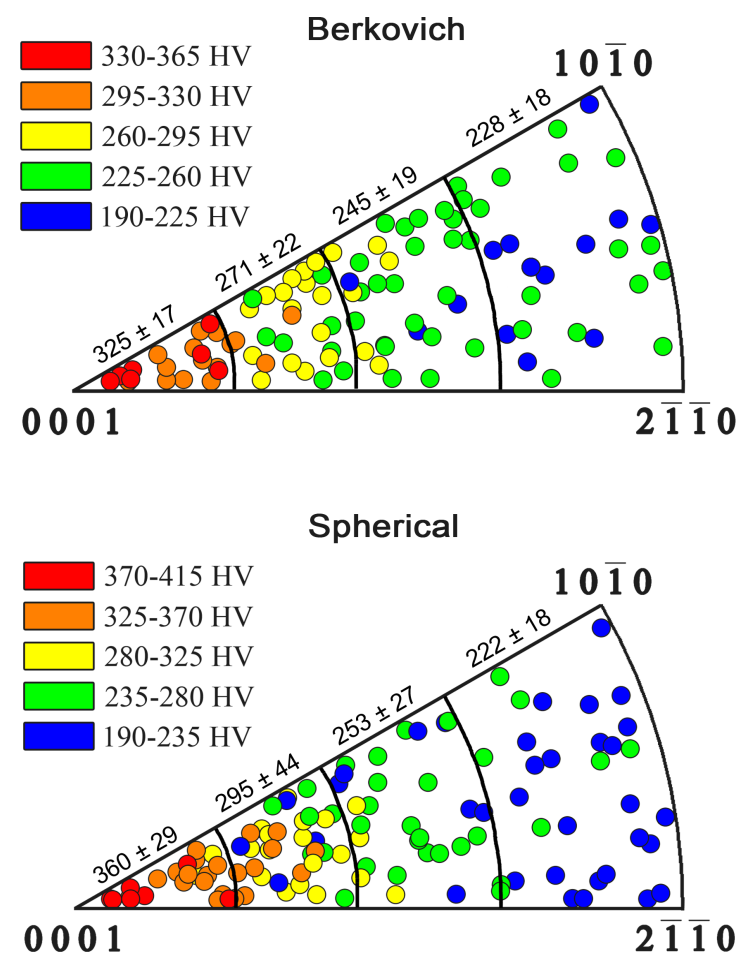

Figure 3. Hardness inverse pole figures (HIPF) with both spherical and Berkovich tips. 